Review

\title{
Practical Guide to Ultrasound Beam Forming: Beam Pattern and Image Reconstruction Analysis
}

\author{
Libertario Demi \\ Department of Information Engineering and Computer Science, University of Trento, 38123 Trento, Italy; \\ libertario.demi@unitn.it
}

Received: 9 August 2018; Accepted: 1 September 2018; Published: 3 September 2018

\begin{abstract}
Starting from key ultrasound imaging features such as spatial and temporal resolution, contrast, penetration depth, array aperture, and field-of-view (FOV) size, the reader will be guided through the pros and cons of the main ultrasound beam-forming techniques. The technicalities and the rationality behind the different driving schemes and reconstruction modalities will be reviewed, highlighting the requirements for their implementation and their suitability for specific applications. Techniques such as multi-line acquisition (MLA), multi-line transmission (MLT), plane and diverging wave imaging, and synthetic aperture will be discussed, as well as more recent beam-forming modalities.
\end{abstract}

Keywords: medical ultrasound; beam forming; ultrasound imaging; multi-line acquisition; multi-line transmission; plane wave; diverging wave; synthetic aperture; parallel beam forming; beam pattern; image reconstruction

\section{Introduction}

In ultrasound medical imaging, beam forming in essence deals with the shaping of the spatial distribution of the pressure field amplitude in the volume of interest, and the consequent recombination of the received ultrasound signals for the purpose of generating images. One can thus navigate through the different techniques using the following question as a compass: which imaging features are important to my application of interest, and which features can I sacrifice? There is, in fact, no ultimate beam-forming approach, and the answer to the previous question strongly depends on what one wants to see in the images.

Below are the key imaging features that will be considered in this paper to review the different beam-forming techniques, along with their descriptions:

Spatial resolution: the smallest spatial distance for which two scatterers can be distinguished in the final image. Spatial resolution can be either axial (along the direction of propagation of the ultrasound wave), lateral, or elevation resolution (along the plane to which the direction of propagation is perpendicular). This feature is normally expressed in $\mathrm{mm}$.

Temporal resolution: the time interval between two consecutive images. This feature is normally expressed in $\mathrm{Hz}$.

Contrast: the capability to visually delineate different objects, e.g., different tissue types, in the generated images. This feature is generally expressed in $\mathrm{dB}$, and it is a relative measure between image intensities.

Penetration depth: the larger depths for which a sufficiently high signal-to-noise ratio (SNR) level can be maintained. This feature is normally expressed in $\mathrm{cm}$.

Array aperture: the physical sizes of the surface representing the combined distribution of active and passive ultrasound sensors: in other words, the array footprint. The array aperture is defined 
by the number of ultrasound sensors (elements), their sizes, and their distribution. This feature is generally expressed in $\mathrm{cm}^{2}$.

Field of view (FOV): the sizes of the area represented by the obtained images. This feature is generally expressed in $\mathrm{cm}^{2}$ or $\mathrm{cm}^{3}$.

Although introduced individually, these features are strongly related. For example, a decrease in temporal resolution can be traded to achieve a higher spatial resolution or a larger FOV; a deeper penetration depth can be achieved by lowering the transmitted center frequency, thus deteriorating spatial resolution, or a broader insonification area can be achieved by widening the transmitted beam, which will result in lower pressure levels being generated, thus lowering the SNR compared to a focused beam. To help the reader become more familiar with these concepts, a simple model can be used. Assuming linear propagation, the following wave equation can be applied to model the pressure field generated by an arbitrary source which propagates in a homogeneous medium [1]:

$$
\partial_{x}^{2} p(x, t)-\frac{1}{c_{0}^{2}} \partial_{t}^{2} p(x, t)=S(x, t)
$$

Here, $\partial_{x}^{2}$ and $\partial_{t}^{2}$ represent the second-order derivative as regards space and time, respectively, $p(x, t)$ is the pressure field, $t$ is time, $x=(x, y, z)$ is the three-dimensional spatial coordinate in a Cartesian system, $c_{0}$ is the small signal speed of sound, and $S(x, t)$ is the source. For a monochromatic point source, i.e., $S(x, t)=\delta(x) \cos \left(2 \pi f_{0} t\right)$, the solution to this equation is known [1], and can be expressed as:

$$
p_{M P s}(x, t)=\frac{P_{0}}{4 \pi|x|} \cos \left[2 \pi f_{0}\left(t-\frac{|x|}{c_{0}}\right)\right]
$$

In this equation, $p_{M P s}(x, t)$ is the pressure field generated by the monochromatic point source, $P_{0}$ is the source amplitude, and $f_{0}$ is the source frequency. This solution is useful, because the pressure field generated by every source can be approximated as the sum of the pressure generated by several point sources, the position of which models the actual shape of the source. The following equation can then be applied:

$$
p(x, t)=\sum_{i=1}^{N} \frac{P_{0}}{4 \pi D_{i}} \cos \left[2 \pi f_{0}\left(t-\frac{D_{i}}{c_{0}}\right)\right]
$$

with $N$ being the number of point sources, $D_{i}$ being the distance between the point for which the pressure field is calculated, and the source being $i$. Equation (3) can also be expressed in its complex formulation as follows:

$$
p(x, t)=\sum_{i=1}^{N} \frac{P_{0}}{4 \pi D_{i}} e^{-j 2 \pi f_{0}\left(t-\frac{D_{i}}{c_{0}}\right)}=P_{0} e^{-j 2 \pi f_{0} t} \sum_{i=1}^{N} \frac{e^{j 2 \pi f_{0} \frac{D_{i}}{c_{0}}}}{4 \pi D_{i}} \propto \sum_{i=1}^{N} \frac{e^{j 2 \pi f_{0} \frac{D_{i}}{c_{0}}}}{4 \pi D_{i}} .
$$

The maximum pressure at a given location is thus obtained when the distances $D_{i}$ are all the same, i.e., the point sources are placed on the surface of a sphere with radius $r$ and centered at the location where the pressure field is calculated. Alternatively, in a case where the sources cannot be arranged in that way, each source could be multiplied by a phase coefficient that compensates for the differences between each term $D_{i}$. In essence, this means time delaying the source according to its distance from the point where the pressure field is calculated. Moreover, to increase the pressure field amplitude, one can increase the physical size of the actual source (the aperture), which entails increasing the number of point sources that are needed to describe it. From Equation (4), we can thus conclude that by applying appropriate phase coefficients, we can maximize the pressure field generated by an arbitrarily shaped source, and that the larger the aperture, the higher the pressure field. Generating high amplitudes means improving the signal strength, and thus the SNR. 
If we then associate a specific phase and amplitude to each source, and model this as a function of the spatial coordinates $A(\boldsymbol{x})$, we can reformulate Equation (4) as:

$$
p(x, t)=e^{-j 2 \pi f_{0} t} \sum_{i=1}^{N} \frac{A(x)}{4 \pi D_{i}} e^{j 2 \pi f_{0} \frac{D_{i}}{c_{0}}}
$$

If we then assume that the source lies on the plane $z=0$, and that the point with coordinates $x=(x, y, z)$ lies on a plane parallel to the plane $z=0$, and at distance $L$ from it, with $L$ being much larger than the maximum distance between two point sources inside the planar surface representing the actual source, then we can write:

$$
D_{i}=\sqrt{L^{2}+(x i-X)^{2}+\left(y_{i}-Y\right)^{2}}
$$

with $\left(x_{i}, y_{i}\right)$ being the coordinates of each point source, and $(X, Y)$ being the coordinates describing the point $x$ on the plane parallel to the plane $z=0$. Using a binomial expansion, Equation (6) can be rewritten as:

$$
D_{i}=L\left(1+\frac{x_{i}^{2}+X^{2}-2 x_{i} X+y_{i}^{2}+Y^{2}-2 y_{i} Y}{2 L^{2}}\right)
$$

and assuming $L \gg X, Y \gg x_{i}, y_{i}$ we can approximate:

$$
D_{i}=L\left(1+\frac{X^{2}-2 x_{i} X+Y^{2}-2 y_{i} Y}{2 L^{2}}\right)
$$

Combining Equation (8) with Equation (5) we obtain:

$$
p(X, Y, t)=\frac{e^{-j 2 \pi f_{0} t} e^{\frac{j 2 \pi f_{0}}{c_{0}} L\left(1+\frac{X^{2}+Y^{2}}{2 L^{2}}\right)}}{4 \pi L} \sum_{x} \sum_{y} A(x, y) e^{-\frac{j 2 \pi(x X+y Y)}{\lambda_{0} L L}}
$$

with $A(x, y)=0$ where there is no source. Thus, the pressure field is proportional to the two-dimensional discrete Fourier transform of the function describing the source. Note that we have approximated $D_{i}$ with $L$ as regards the amplitude term inside the summation in Equation (5). This was not the case for the phase term. In fact, in this case also, small variations of $D_{i}$ with respect to $\lambda_{0}=\frac{c_{0}}{f_{0}}$ can be significant. From Equation (9), we can deduce that for a circular aperture with radius $R$, we can write the pressure field as:

$$
\left.p(X, Y, t)\right|_{Y=0} \propto \sin c\left(\frac{R X f_{0}}{c_{0} 2 L}\right)
$$

From Equation (10), we can deduce that the ultrasound beam size is influenced by the aperture size and transmitted frequency, and that it changes over depth. The beam can be defined as the area where the pressure amplitude is above a specific value, which is normally considered in relation to the maximum pressure generated (e.g., the $-20 \mathrm{~dB}$ beam). A larger aperture and higher frequencies mean a smaller beam. Moreover, the beam generally widens with increasing depths. The beam size defines the spatial resolution in the lateral and elevation direction. The smaller the beam, the higher the spatial resolution. On the other hand, the smaller the beam, the smaller the volume that can be insonified with a single transmission, and more transmission events are thus required to cover a given volume. In the next section, the basic differences between linear and phased array beam forming will be introduced. Subsequently, multi-line acquisition (MLA), multi-line transmission (MLT), plane and diverging wave imaging, synthetic aperture, and more recent beam-forming modalities will be described. To summarize the analysis, a table is presented where the peculiarities of each modality are highlighted. 


\section{Linear and Phased Array Beam Forming}

We can start by describing the source that generates the ultrasound fields. In particular, we will address its aperture and how we could excite it by means of electrical signals. As represented in Figure 1, ultrasound sensors, which are generally able to both transmit and receive ultrasound signals, can be arranged so that their centers cover a surface, a line, or a curve. In the first case, we have a matrix or two-dimensional (2D) array, while in the second and third case, we have a one-dimensional (1D) array. The distance between the centers is referred to as pitch, and the size of the empty space between consecutive sensors is called kerf [2,3]. For 2D arrays, the pitch and kerf may be different along the lateral $(\mathrm{x})$ and elevation $(\mathrm{y})$ directions. In general, sensors do not need to be arranged in a periodic structure. In fact, an aperiodic sensor distribution can produce benefits such as the reduction of the effects of side lobes [4].

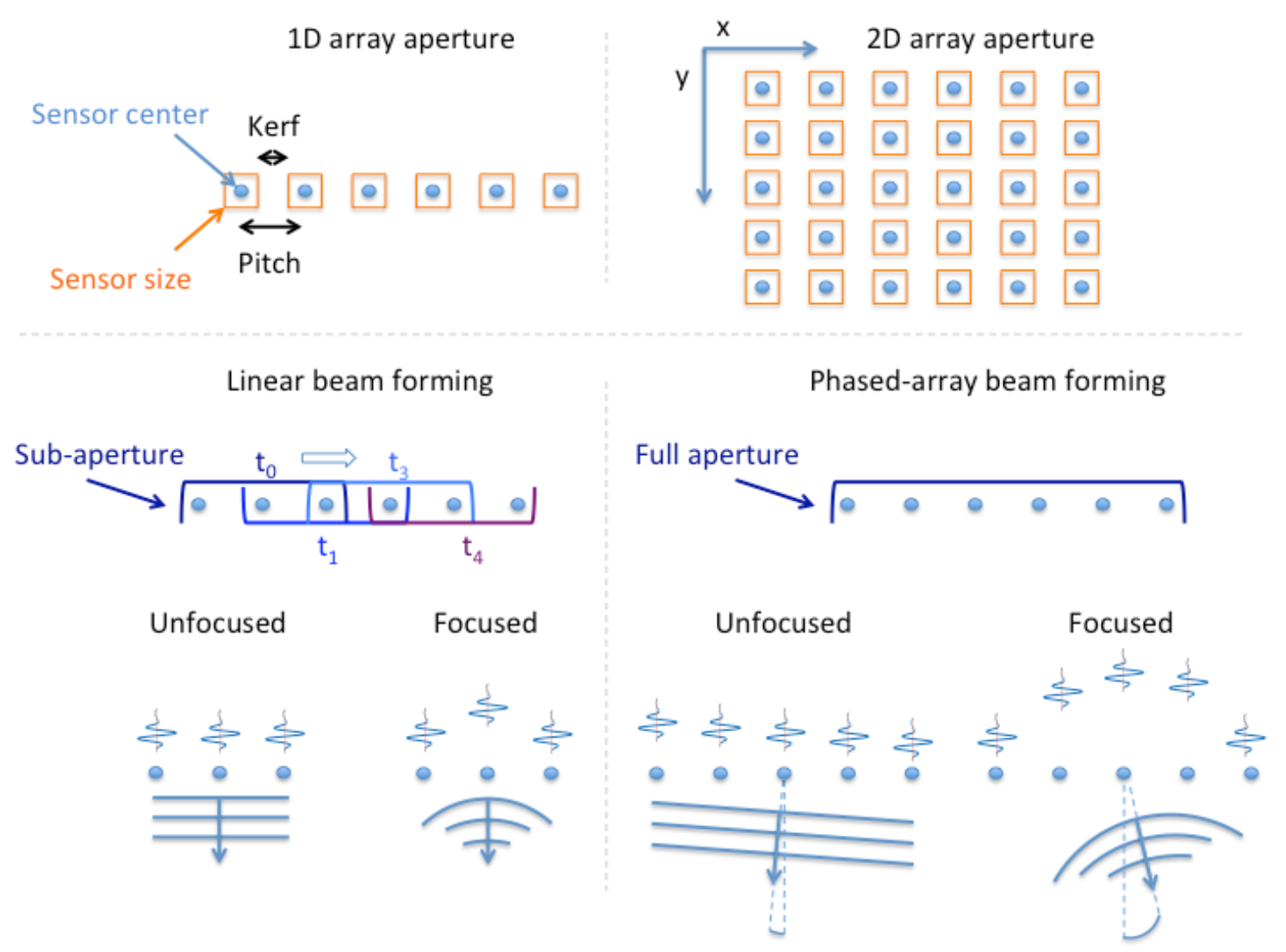

Figure 1. This figure shows the different sensor distributions for a one-dimensional (1D) and twodimensional (2D) array aperture (top), together with an overview of the possible driving schemes for linear and phased array beam forming (bottom) in cases with a focused and an unfocused beam.

In principle, as described for point sources, each sensor can be excited by a signal having its own amplitude, phase, and waveform. However, sensors are generally grouped in sub-apertures, and within one sub-aperture, the same waveform is transmitted, but with a different phase and amplitude.

This is true for linear array beam forming, where a sub-aperture is defined and used both to transmit and receive ultrasound fields. The signal so acquired is then representative of the structures seen by the ultrasound waves over depth and in front of the sub-aperture. This signal is called an A-scan [2,3]. Subsequently, this sub-aperture is linearly shifted over the entire array so as to obtain multiple A-scans, ultimately forming an image line by line. The sensors that belong to a sub-aperture could be excited by signals that share the same phase, i.e., the unfocused case, or have different phases, as in the focused case. As can be deduced from Equation (4), when focusing is applied, higher 
pressures are generated. Moreover, smaller beams may even be achieved. Thus, focusing implies that the spatial (in the lateral and/or elevation direction) resolution, SNR, and penetration depth are improved. On the other hand, the area investigated by every beam is smaller, which means that more beams are necessary to cover a given FOV compared to the unfocused case. This also implies that more transmission events are required to form an image, which may decrease the frame rate. Within a given sub-aperture, the sensors could also be excited with different amplitudes. This is true if an apodization mask is used. Using an apodization mask reduces the amplitude of side lobes and their effects on the final image, but negatively affects the lateral and/or elevation spatial resolution. Furthermore, the maximum pressure generated is reduced, and thus consequently so are the SNR and penetration depths [2,3].

Unlike linear array beam forming, with phased array beam forming, the entire array aperture is used for each transmission. The phases of the driving signals are specifically adjusted for every sensor at each transmission event so as to steer the beam, and place it at a given angle with respect to the direction that is normal to the array aperture [2,3]. Different sets of phases are then used to obtain different steering directions, produce multiple A-scans, and thus form an image. With phased array beam forming, the beam could be a focused or unfocused beam, and apodization could be used. It is important to add that a particular constrain is present for phased array beam forming: the pitch has to be smaller than half the wavelength in order to avoid grating lobes. These are additional lobes, which can further degrade the image quality [2,3]. In Figure 1, a schematic overview of what has been introduced in this section is presented. Linear and phased array beam forming strategies are represented only for a 1D aperture, but these can of course be also applied to a 2D aperture, which gives more flexibility in the definition of the sub-apertures. Moreover, with a 2D aperture, the beam can be steered through the entire volume, rather than only on a plane perpendicular to the aperture [2,3].

When comparing linear and phased array beam forming, a list of pros and cons can be made. Both approaches form an image line by line, with one line being generated at every transmission event. Linear arrays can image only the area in front of the aperture, while a larger area can be imaged with phased arrays as the beam can be steered. This also means that the aperture of a linear array has to cover the entire area of interest (along the lateral direction). However, this is not the case for phased arrays. Consequently, phased arrays are particularly suitable in situations where there is a small imaging window, as in transthoracic ultrasound imaging, where the ribs represent an obstacle for imaging [5,6]. On the other hand, the geometries of phased arrays are constrained by the phenomenon of grating lobes, which is particularly demanding when using high frequencies. As a result, more accurate phase sets, and as many as the amount of steering angles, are required.

The transmit phase (or active phase), which is the phase that defines the shaping of the spatial distribution of the pressure field amplitude in the volume of interest, has been our focus thus far. In the receive phase, the very same phase sets and apodization functions that are used in the transmit phase can be applied. However, the received echo signals can be also treated differently. Furthermore, a different group of elements than those used in the transmission phase can also be used, as is, for example, the case for synthetic aperture beam forming $[7,8]$ and multi-line acquisition beam forming [9]. Figure 2 illustrates the differences in the spatial distribution of the pressure amplitudes. The $-20 \mathrm{~dB}$ beams obtained with a focused sub-aperture, and with an unfocused, focused, and steered full-aperture, are shown. The typical FOVs achievable with linear and phased array beam forming are also shown. Note that when the linear array sensors are distributed along a curve, a larger field of view can also be obtained. This is the case with convex probes. However, the probe loses its flat surface [10]. 
Sub-aperture Focused
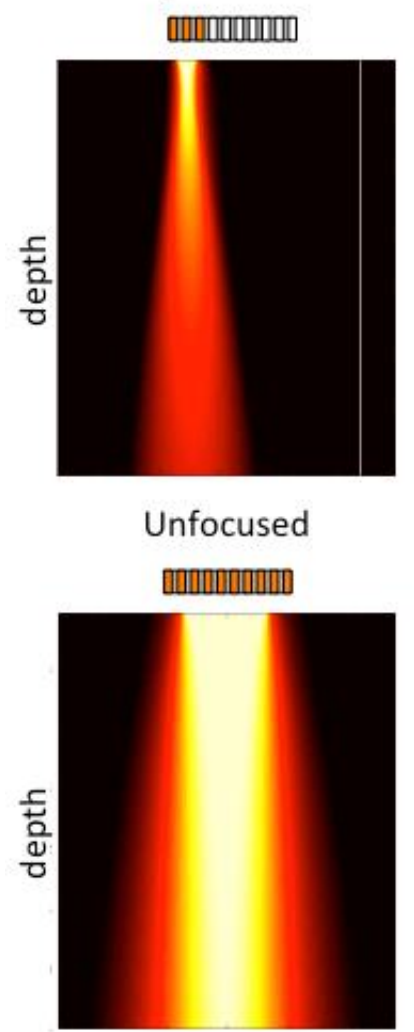

lateral
Linear BF FOV
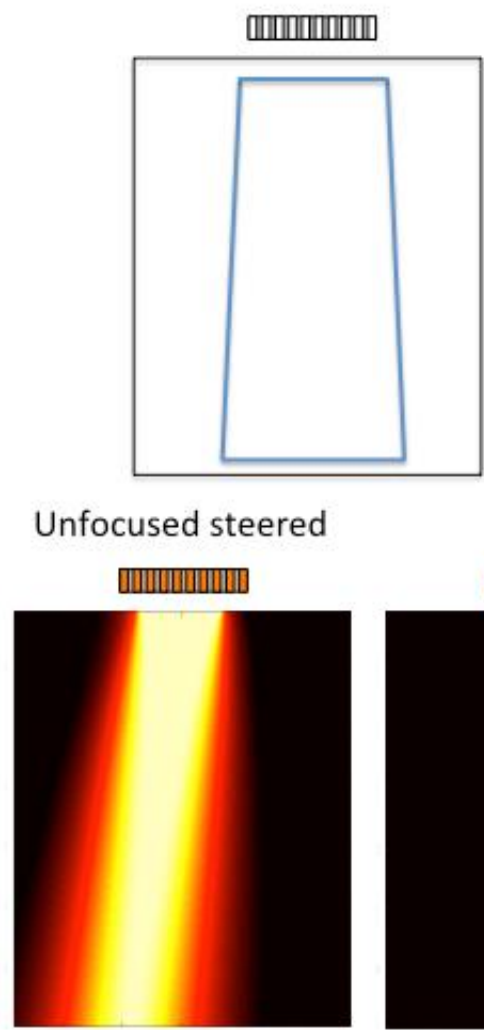

lateral

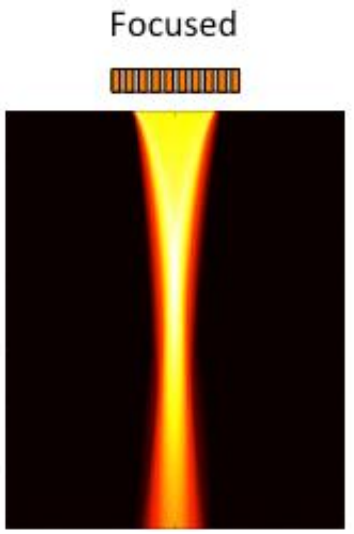

lateral
Phased BF FOV

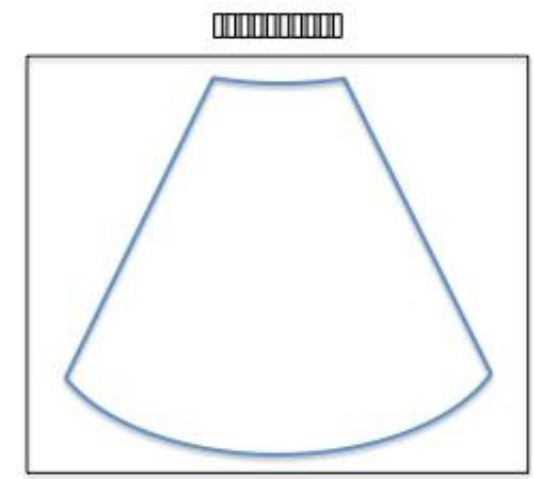

Focused steered

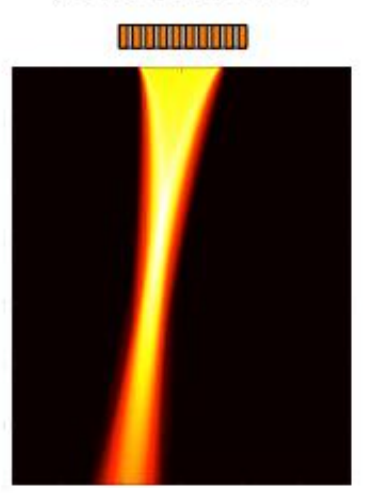

lateral

Figure 2. This figure shows the different spatial distribution of pressure amplitudes when unfocused, focused, and steered beams are generated. Moreover, the typical field of view (FOV) that is achievable with linear and phased array beam forming is also shown. These beam profiles were generated using the software package k-Wave [11].

\section{Multi-Line Acquisition and Multi-Line Transmission Beam Forming}

As briefly introduced above, the beam does not need to be the same in the transmit and receive phase. This is certainly the case with multi-line acquisition (MLA) beam forming. The basic idea behind this approach is to transmit a wide beam, so that a large area is covered, and then make use in receive of multiple, narrower beams, in order to form several A-scans along different directions for each transmission event. In this way, multiple lines are formed in parallel, thus increasing the frame rate and improving the temporal resolution. The receive phase is in fact defined by how the different signals received by all of the array elements are combined to form a line of the image. Therefore, it is possible to apply different phase sets and apodization masks to the signals received after a single transmission event, thus allowing the formation of multiple lines in parallel. In fact, these techniques are also referred to as parallel receive beam forming. In Equation (10), we can see that a wider beam can be achieved by using, without focusing, a small sub-aperture at the center of the array during transmission [9,12-14]. Since not all of the elements are utilized, and as a result the active aperture is reduced in transmit, the maximum pressure generated is consequently lower compared to the case where all of the elements are used. Furthermore, the spatial resolution (although not in the axial direction) is not as good, as focusing is applied only in the receive phase. Not only can MLA be applied to achieve a gain in the frame rate, it can also be applied to improve the SNR and contrast by simply averaging consecutive images obtained at a higher temporal resolution than with standard beam forming (i.e., techniques where only one line is generated per each transmission event). 
Moreover, MLA techniques can also be used to image a larger FOV. In this case, the gain in acquisition rate is used to widen the area covered by the imaging system. To summarize with a simple example, in case 4, image lines could be formed in parallel, which means that: (a) the temporal resolution could be improved by factor of four, or (b) four consecutive images could be averaged to improve the SNR, or (c) a FOV that is four times larger could be in principle imaged, or (d) a combination of these gains could be achieved by spending the higher data acquisition rate in the most desirable way (e.g., averaging only two consecutive images and thus improving the SNR while still improving also the frame rate by factor of two).

A similar concept could be also applied at inverted phases: instead of having parallel lines being formed in the receive phase, they could be generated during transmission. This approach is referred to as multi-line transmission (MLT) or parallel transmit beam forming. Even in the case where the very same phase sets and apodization functions are used, and are simply swapped between the transmit phase and the receive phase, advantages can already be obtained. This is the case for tissue harmonic imaging applications. Unlike standard (fundamental) ultrasound imaging, this modality makes use of the harmonic components that are generated during ultrasound propagation, and not the pressure fields directly emitted by the array, to form an image. The harmonic components represent a part of the pressure wave fields, which is located around multiples of the transmitted center frequency. For a pulse-echo imaging system, an improved spatial resolution, a reduction of reverberation, grating, and side-lobe artifacts [15] are among the advantages of utilizing tissue harmonic imaging. In particular, MLT beam forming is better than MLA when applied to tissue harmonic imaging, because the higher pressure amplitude that is generated - thanks to a focused beam in transmission-is fundamental to boost the generation of sufficiently strong harmonic components. When applied to harmonic imaging, MLT beam forming provides a further reduction of the side-lobe amplitudes and an increase in SNR [16].

To generate multiple beams in transmission, different approaches are possible. One approach is to simply distribute multiple focused beams in the volume of interest. This is achieved by a linear superposition of the signals that are used to generate each individual beam. As a side effect, this limits the maximum signal strength that is applicable to the formation of every beam, and thus lowers the maximum pressure that can be generated by a single focused beam [17]. The MLT approach can be used both with linear array and phased array beam forming, and with 1D and 2D arrays [17-19]. To minimize the possible inter-beam interference generated by neighboring transmitted beams, specific sets of apodization functions can be applied [20]. Additionally, another approach to reduce the interbeam interference is to separate the different beams in the frequency domain. With this approach, which is referred to as frequency division multiplexing, the available transducer bandwidth is divided into orthogonal sub-bands, each of which is allocated to a beam. Multiple beams, as many as the number of sub-bands, can thus be transmitted in parallel, and the generated echo signals can then be identified in the receive phase by means of band-pass filters [21]. The main disadvantage of this method is the loss in axial resolution due to the subdivision of the available band into smaller sub-bands. A smaller sub-band implies, in fact, a longer pulse.

In general, when implementing MLT, it is beneficial to add small time delays between the signals that are used to generate multiple beams in transmission. This improves their capability to separate the different beams. As a side effect, it lengthens the transmit phase, and thus increases the depth for which the final imaging system will be blind $[17,18]$. With MLT, the inter-beam interference level required by the specific application of interest limits the number of parallel beams. In general, a higher number of parallel beams results in a higher level of interference [18-20].

Figure 3 illustrates the effect of inter-beam interference on the final image. An ultrasound image of four wire targets obtained by linear array beam forming is shown (top left corner). The same targets are then imaged using MLT applied to linear array beam forming and performed by frequency division multiplexing, with three beams in transmission (top right corner). As can be seen, four MLT "ghost" wires appear before and in front of each "actual" wire. This type of artifact is also present when MLT 
is performed by spatially distributing the transmitted beams over the volume of interest. However, the location of the artifact is different. To illustrate this phenomenon, a single wire is imaged using MLT without frequency division multiplexing with six beams in transmission (bottom). In this case, the image was obtained by MLT applied to phased array beam forming. Ghost wires are visible on the sides of the actual wire.

As for MLA, and also with MLT, the higher data acquisition rate achieved by generating multiple beams in transmission is not solely applicable to improve the frame rate. For example, when implementing MLT by means of orthogonal frequency division multiplexing, a multi-focusing imaging approach can be realized where the different sub-bands are used to generate beams with a focus at different depths. In particular, the lower the center frequency of the sub-band, the deeper the focus will be. Thanks to this approach, the penetration depth and signal-to-noise ratio (SNR) improves without affecting the frame rate [22]. In conclusion, it is important to note that MLA and MLT techniques can be combined together to have a multiplicative effect on the gain in the data acquisition rate [21,23].
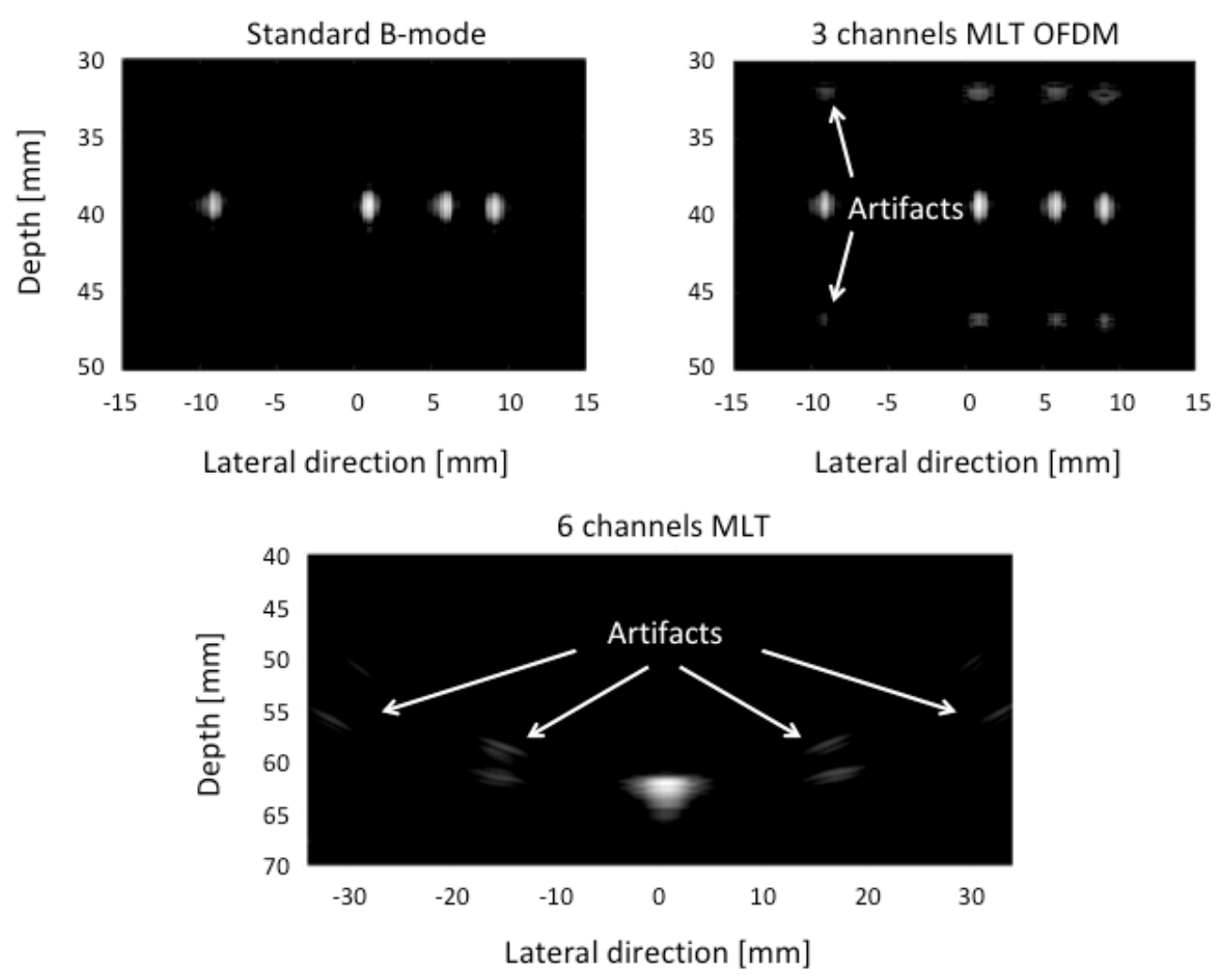

Figure 3. This figure illustrates the effect of inter-beam interference on the image. A standard linear array beam forming image of four wires is shown on the top left corner. The same wires are then imaged with multi-line transmission (MLT) performed by frequency division multiplexing, and applied to linear array beam forming. The corresponding image is shown in the top right corner, and "ghost" wires are clearly visible before and after each wire. At the bottom, an image of a single wire obtained with MLT performed without frequency division multiplexing, and applied to phased array beam forming, is shown. Also in this case, ghost wires are visible, but at a different position relative to the actual wire.

\section{Plane and Diverging Wave Beam Forming}

With these techniques, the emphasis is certainly more on improving the achievable frame rate. Plane wave imaging originates from studies aimed at imaging the transient propagation of shear 
mechanical waves in real time [24-26]. For this type of application, a frame rate in the order of thousands of frames per second is needed. The basic concept is that if one can reduce the number of transmission events that are needed to form an image to the bare minimum, this implies maximizing the frame rate. The absolute minimum is of course one transmission event per image. In this case, the frame rate is, in essence, only limited by the speed of the ultrasound wave in the imaging medium, the depths that need to be visualized, and the processing time that is necessary to form the image. Thus, the idea is to approximate in transmission the generation of a plane wave, achieving, in this way, a very wide and homogeneous beam (as wide as the array aperture). This can be achieved by simply exciting all of the transducer elements with the same phase for each transmission event. Then, in the receive phase, the signals acquired by all of the elements are processed with different phase sets and amplitudes, and multiple lines are generated in parallel. In particular, all of the lines that form an image are generated with the echo signals, which are received after a single transmission. In simple words, this approach can be seen as a radical MLA approach where only one large beam is used in transmission. As in the case of MLA, this technique suffers from lower pressure amplitudes being generated compared to focused beams, thus affecting the SNR and penetration depths. Moreover, the straightforward approach of plane wave imaging suffers from low image quality in terms of spatial resolution and contrast [27]. In fact, all of the imaging features are sacrificed to maximize the temporal resolution. A way to balance the performance of plane wave imaging among the different imaging features is to compromise, or in other words, to apply image compounding. Compounding essentially means averaging. However, it is not a simple averaging of consecutive frames. With plane wave coherent compounding, steering is applied, and thus the "plane wave" is no longer propagating only straight in front of the transducer array, it is also propagating under a given angle [27]. An image is then formed for varying transmission angles, and in the end, averaging is performed over the images obtained with all of the different angles. In this way, the gain in frame rate is reduced by a factor that is equal to the number of angles. On the other hand, the other imaging features (spatial resolution, SNR, penetration depth, contrast) are improved. However, in order to obtain a performance that is comparable to standard beam forming, the amount of compounded angles is very high (in the order of 70), and the extreme gain in frame rate that is achievable with plane wave imaging is substantially lost $[27,28]$. The number of angles, as well as the maximum steering angle, can be adjusted to tune plane wave imaging for a specific application. However, the most important feature of this technique is its capability to reach really high frame rates, which makes it extremely suitable for applications where fast phenomena need to be observed. In this situation, the spatial resolution is actually less important, while the key feature is the temporal resolution. Shear wave imaging is certainly a good example $[29,30]$. Other interesting areas of application are flow, contrast dynamics, and functional ultrasound imaging [31]. It is also important to mention that the implementation of this high frame rate imaging method has also been made possible thanks to the developments of GPU technologies, which provide the computational speed that is required to process the amount of data generated during plane wave imaging [32-34].

Diverging wave beam forming does not differ substantially from plane wave imaging. The small difference between the two methods lies in a defocused beam being used in transmission during diverging wave beam forming, which allows for an even larger insonification area [28,35-37]. Multiple parallel lines are generated in the receive phase in this case also, and compounding algorithms can be applied. In general, the same considerations as in plane wave imaging apply. Neither plane wave or diverging wave imaging are ideal for applications where a small array aperture is required, as is the case in transthoracic ultrasound imaging, where the presence of the ribs constrain the size of the imaging window. Moreover, due to the low-pressure amplitudes that are generated in the transmit phase, these techniques are certainly not ideal for tissue harmonic imaging applications, where high-pressure values are needed in order to generate the harmonic components that are necessary to form the image $[16,38,39]$. 


\section{Synthetic Aperture Beam Forming}

Synthetic aperture is a beam-forming approach that originates from the world of radar and was first implemented for medical ultrasound imaging in the late 1960s and early 1970s [40,41]. In its basic implementation, only one element is excited for every transmission event [42]. In the receive phase, all of the elements of the array are used to receive the echo signals, and a low quality image is generated for every transmission event. The key aspect is that every point of the image is obtained by taking into account the geometrical distance between each transmitting element and each receiving element. Thus, assuming a constant speed of sound through the imaging volume, appropriate phase sets are used to compensate for the differences in the arrival time. Subsequently, the received time-compensated signals are added together.

As a result, the images that are obtained for each transmission are combined to obtain an image of higher quality in terms of spatial resolution, contrast, and penetration depth with respect to the images obtained for every transmission. Thus, focusing is performed for every pixel in the image, and applied both in the transmit phase (indirectly by recombining the images formed with a single emitter) and the receive phase. As a consequence, the highest possible spatial resolution for delay-and-sum beam forming is obtained everywhere in the image [43]. However, the signal-to-noise ratio and penetration depths are significantly degraded by the array aperture being minimized in transmission, since only one element is active. Transmitting with sub-apertures rather than with a single element can mitigate this phenomenon [44-46]. However, the accuracy in the image reconstruction given by the availability of the data as obtained from the entire transmitting-receiving pairs of elements is lost, which deteriorates the spatial resolution. Once again, improving the performance with respect to a given imaging feature implies accepting that there will be a loss in performance with respect to another.

An interesting approach based on frequency division multiplexing has been proposed to improve the SNR and penetration depth without losing access to the full element-to-element data set [47]. Similarly to the case discussed for MLT, the available transducer bandwidth is divided into sub-bands. During each transmission, all of the elements are active, with each operating at one specific sub-band. During consecutive transmissions, every element is active at a different sub-band, and the entire bandwidth is covered. In the receive phase, band-pass filters are used to separate and identify the signal coming from the different elements. Using this approach, the entire aperture is active for every transmission event, and the achievable SNR and penetration depth are thus improved. Another possibility is to use chirp signals in transmission, and a matched filter in the receive phase. Particular attention to the signal properties is needed when chirp signals are used, so as to avoid temporal side lobes. Furthermore, additional processing steps and the ability of the hardware to generate well-controlled electrical signals is also required. However, this approach can improve penetration depth and axial resolution [48-50]. It is also important to note that if only one element is active for every transmission event, this implies that the time that is needed to collect all of the signals necessary to form an image is maximized, which in other words means minimizing the frame rate. However, not all of the elements of the array need to be used in transmission. In this way, a higher frame rate can be achieved. On the other hand, this will lower the spatial resolution and increase the amplitude of the side-lobes and their effect on the final image [51].

\section{Comparison among Different Beam-Forming Options}

A general comparison between the different techniques discussed thus far can be drawn. Figure 4 illustrates the different driving schemes for MLA, MLT, plane wave, diverging wave, and synthetic aperture beam forming. The transmit beams are represented in orange, and different shades of orange are used to highlight the multiple beams for MLT, and separate the beam profiles for plane and diverging wave beam forming, respectively. For MLA, the receive beams are also shown in shades of blue. The duration of the transmit phase is also emphasized for MLT. Table 1 summarizes the peculiarities of each modality. A comparison is made with standard line-by-line beam forming. A plus or minus sign means that the performance with respect to that specific imaging feature (one for each 
column) is improved or reduced, respectively. MLA and MLT beam forming is essentially trading spatial resolution for data acquisition rate, and can be generally applied to any array aperture. In the case of MLT especially, where focused beams are used in transmission, penetration depth is not lost compared to standard beam forming. Plane and diverging wave beam forming focus instead on achieving a very high data acquisition rate. Consequently, spatial resolution and penetration depth are affected. Moreover, these approaches substantially require a large aperture size. This is also true for synthetic aperture beam forming, where an increase in the number of transmitting elements leads to improved performance. The strength of synthetic aperture beam forming is certainly on the attainable spatial resolution, which is achieved at the expense of penetration depth and the data acquisition rate.

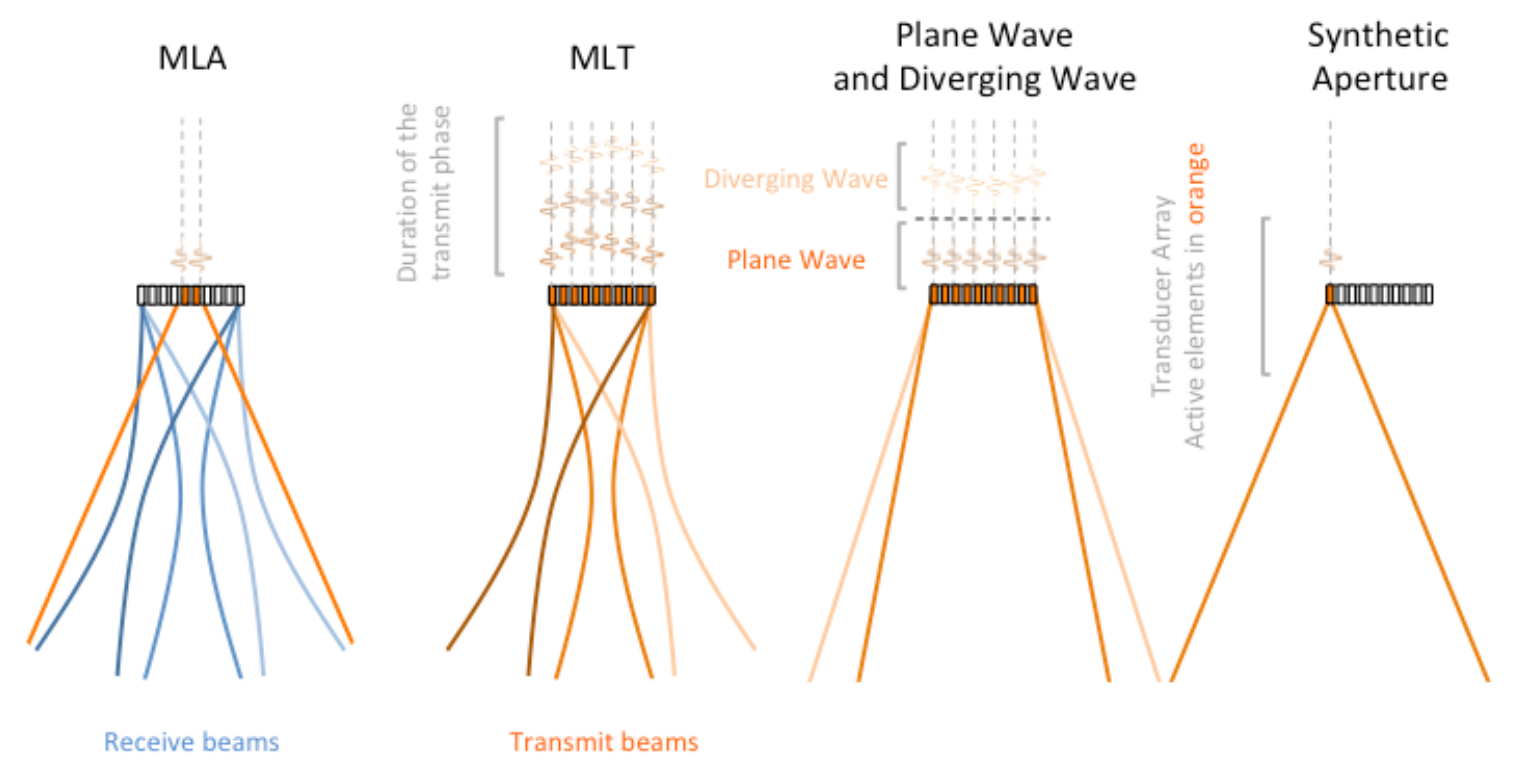

Figure 4. This figure illustrates the different driving schemes for MLA, multi-line transmission (MLT), plane wave, diverging wave, and synthetic aperture beam forming. The transmit beams are represented in orange, and different shades of orange are used to highlight multiple beams for MLT, and separate the beam profiles for plane and diverging wave, respectively. For MLA, the receive beams are also shown in shades of blue. The duration of the transmit phase is also emphasized for MLT.

As described in the previous sections, for every technique, these pros and cons can be mitigated by specific implementations. However, the rule that what one gains regarding particular feature implies a loss in performance with respect to the other features generally applies.

Table 1. This table summarizes the peculiarities of each modality. A plus or minus sign means that the performance with respect to that specific imaging feature (one for each column) is improved or reduced, respectively. The 0 sign means that there is no significant variation. The evaluation is performed with respect to standard beam-forming performance.

\begin{tabular}{ccccc}
\hline Beam Forming Strategy & Spatial Resolution & Data Acquisition Rate & Array Aperture Size & Penetration Depths \\
\hline MLA-MLT & - & + & 0 & 0 \\
Plane and Diverging Wave & - & ++ & - & - \\
Synthetic Aperture & + & - & - & - \\
\hline
\end{tabular}

\section{Other Beam-Forming Strategies}

Several emerging beam forming strategies, besides those dealt with in this paper, have been reported and discussed in the literature, including approaches based on machine learning [52]. Particularly interesting concepts are those explored with null subtraction imaging (NSI), and coherence beam forming. With NSI, particular sets of apodization functions are used to achieve a lateral 
(and potentially elevation) spatial resolution that goes beyond the diffraction limit. This technique requires the application of signal-processing techniques only in the receive phase. In essence, the idea is to combine the images formed using zero mean and non-zero mean apodization functions. A zero-mean apodization generates a beam with a "hole" along the beam axis (see Huygens' principle). Consequently, this beam can be subtracted from that generated by a non-zero mean apodization function, thus obtaining an extremely sharp beam. However, the gain in spatial resolution is costly in terms of contrast [53-55].

When using coherence beam forming, each imaging pixel is obtained from the integration of the normalized covariance matrix that is calculated between the signals received by all of the elements forming the array [56]. This follows after appropriate time-delay compensations are applied. This technique is clearly more computationally expensive compared to standard beam forming, and generally results in a smaller dynamic range. However, it is particularly suitable for applications in low SNR imaging conditions. Contrast is generally improved, and noise is significantly reduced [56-58].

\section{Conclusions}

In this paper, a review of different beam-forming schemes has been presented. Multi-line acquisition, multi-line transmission, plane wave, diverging wave, synthetic aperture, and more recent beam-forming strategies have been introduced. The peculiarities and advantages of these approaches have been compared, and their applicability has been discussed. In conclusion, it is also important to mention that all of these studies would have not been possible without the development of open research platforms, thanks to which the implementation and testing of advanced beam-forming strategies were carried out [59-64].

Funding: This research received no external funding.

Conflicts of Interest: The authors declare no conflict of interest.

\section{References}

1. Demi, L. Modeling Nonlinear Propagation of Ultrasound through Inhomogeneous Biomedical Media. Ph.D. Thesis, Delft University of Technology, Delft, The Netherlands, 2013.

2. Szabo, T. Diagnostic Ultrasound Imaging: Inside Out, 2nd ed.; Elsevier: New York, NY, USA, 2013.

3. Cobbolt, R.S.C. Foundation of Biomedical Ultrasound; Oxford University Press: Oxford, UK, 2006.

4. Ramalli, A.; Boni, E.; Savona, A.S.; Tortoli, P. Density-tapered spiral arrays for ultrasound 3-D imaging. IEEE Trans. Ultrason. Ferroelectr. Freq. Control 2015, 8, 1580-1588. [CrossRef] [PubMed]

5. Ramm, O.T.; Thurstone, F.L. Cardiac imaging using a phased array ultrasound system I System Design. Circulation 1976, 2, 258-262.

6. Ramm, O.T.; Thurstone, F.L. Cardiac imaging using a phased array ultrasound system II Clinical Techniques and Applications. Circulation 1976, 2, 262-267.

7. Norton, S.J. Acoustical holography with an annular aperture. J. Acoust. Soc. Am. 1982, 71, 1169-1178. [CrossRef]

8. Ajay, K.L.; Kassam, S.A. Side-lobe reduction in the ring array pattern for synthetic aperture imaging of coherent sources. J. Acoust. Soc. Am. 1983, 74, 840-846.

9. Shattuck, D.P.; Weinshneker, M.D. Explososcan: A parallel processing technique for high speed ultrasound imaging with linear phased arrays. J. Acoust. Soc. Am. 1984, 75, 1273-1282. [CrossRef] [PubMed]

10. Szabo, T.L.; Lewin, P.A. Ultrasound transducer selection in clinical imaging practice. J. Ultrasound Med. 2013, 32, 573-582. [CrossRef] [PubMed]

11. Treeby, B.E.; Cox, B.T. K-Wave: MATLAB toolbox for the simulation and reconstruction of photoacoustic wave-fields. J. Biomed. Opt. 2010, 15, 021314. [CrossRef] [PubMed]

12. Smith, S.W.; Pavy, H.G., Jr.; von Ramm, O.T. High Speed Ultrasound Volumetric Imaging System Part I Transducer Design and Beam Steering. IEEE Trans. Ultrason. Ferroelectr. Freq. Control 1991, 38, 100-108. [CrossRef] [PubMed] 
13. Von Ramm, O.T.; Smith, S.W.; Pavy, H.G., Jr. High Speed Ultrasound Volumetric Imaging System Part II parallel processing and image display. IEEE Trans. Ultrason. Ferroelectr. Freq. Control 1991, 38, $109-114$. [CrossRef] [PubMed]

14. Snyder, J.E.; Kisslo, J.A.; von Ramm, O.T. Real time orthogonal mode scanning of the heart: A new ultrasonic imaging modality. J. Am. Coll. Cardiol. 1986, 7, 1279. [CrossRef]

15. Demi, L.; Verweij, M.D. Nonlinear Acoustics. In Comprehensive Biomedical Physics; Elsevier: New York, NY, USA, 2014.

16. Demi, L.; Verweij, M.D.; van Dongen, K.W.A. Parallel Transmit Beamforming Using Orthogonal Frequency Division Multiplexing and Applied to Harmonic Imaging-A Feasibility Study. IEEE Trans. Ultrason. Ferroelectr. Freq. Control 2012, 59, 2439-2447. [CrossRef] [PubMed]

17. Santos, P.; Tong, L.; Ortega, A.; Løvstakken, L.; Samset, E.; D’hooge, J. Safety of Multi-Line Transmit beam forming for fast cardiac imaging-A simulation study. In Proceedings of the IEEE International Ultrasonics Symposium, Chicago, IL, USA, 3-6 September 2014; pp. 1199-1202.

18. Demi, L.; Viti, J.; Kusters, L.; Guidi, F.; Tortoli, P.; Mischi, M. Implementation of Parallel Transmit Beamforming Using Orthogonal Division Multiplexing-Achievable Resolution and Interbeam Interference. IEEE Trans. Ultrason. Ferroelectr. Freq. Control 2013, 60, 2310-2320. [CrossRef] [PubMed]

19. Denarie, B.; Bjastad, T.; Torp, H. MemberMulti-line Transmission in 3D with Reduced Cross-talk Artifacts-A Proof of Concept Study. IEEE Trans. Ultrason. Ferroelectr. Freq. Control 2013, 60, 1708-1718. [CrossRef] [PubMed]

20. Tong, L.; Gao, H.; D’hooge, J. Multi-Transmit Beamforming for Fast Cardiac Imaging-A Simulations Study. IEEE Trans. Ultrason. Ferroelectr. Freq. Control 2013, 60, 1719-1731. [CrossRef] [PubMed]

21. Demi, L.; Ramalli, A.; Giannini, G.; Mischi, M. In Vitro and In Vito Tissue Harmonic Imaging Obtained with Parallel Transmit Beamforming by Means of Orthogonal Frequency Division Multiplexing. IEEE Trans. Ultrason. Ferroelectr. Freq. Control 2015, 62, 2030-2035. [CrossRef] [PubMed]

22. Demi, L.; Giannini, G.; Ramalli, A.; Tortoli, P.; Mischi, M. Multi-focus Tissue Harmonic Imaging Obtained with Parallel Transmit Beamforming by Means of Orthogonal Frequency Division Multiplexing. In Proceedings of the IEEE International Ultrasonics Symposium, Taipei, Taiwan, 21-24 October 2015.

23. Tong, L.; Ramalli, A.; Jasaityte, R.; Tortoli, P.; D’hooge, J. Multi-Transmit Beam forming for Fast Cardiac Imaging-Experimental Validation and In Vivo Application. IEEE Trans. Med. Imaging 2015, 33, 1205-1219. [CrossRef] [PubMed]

24. Sandrin, L.; Catheline, S.; Tanter, M.; Hennequin, X.; Fink, M. Time resolved pulsed elastography with ultrafast ultrasonic imaging. Ultrason. Imaging 1999, 21, 259-272. [CrossRef] [PubMed]

25. Sandrin, L.; Catheline, S.; Tanter, M.; Hennequin, X.; Vinconneau, C.; Fink, M. 2D transient elastography. Acoust. Imaging 2000, 25, 485-492.

26. Sandrin, L.; Catheline, S.; Tanter, M.; Fink, M. Shear modulus imaging using 2D transient elastography. IEEE Trans. Ultrason. Ferroelectr. Freq. Control 2002, 49, 426-435. [CrossRef] [PubMed]

27. Montaldo, G.; Tanter, M.; Bercoff, J.; Benech, N.; Fink, M. Coherent Plane-Wave Compounding for Very High Frame Rate Ultrasonography and Transient Elastography. IEEE Trans. Ultrason. Ferroelectr. Freq. Control 2009, 56, 489-506. [CrossRef] [PubMed]

28. Tong, L.; Gao, H.; Fai Choi, H.; D’hooge, J. Comparison of Conventional Parallel Beam Forming with Plane Wave and Diverging Wave Imaging for Cardiac Applications-A Simulation Study. IEEE Trans. Ultrason. Ferroelectr. Freq. Control 2012, 59, 1654-1663. [CrossRef] [PubMed]

29. Bercoff, J.; Tanter, M.; Fink, M. Sonic boom in soft materials: The elastic Cerenkov Effect. Appl. Phys. Lett. 2004, 84, 2202-2204. [CrossRef]

30. Bercoff, J.; Tanter, M.; Fink, M. Supersonic shear imaging: A new technique for soft tissues elasticity mapping. IEEE Trans. Ultrason. Ferroelectr. Freq. Control 2004, 51, 396-409. [CrossRef] [PubMed]

31. Tanter, M.; Fink, M. Ultrafast Imaging in Biomedical Ultrasound. IEEE Trans. Ultrason. Ferroelectr. Freq. Control 2014, 61, 102-119. [CrossRef] [PubMed]

32. So, H.; Chen, J.; Yiu, B.; Yu, A. Medical ultrasound imaging: To GPU or not to GPU? IEEE Micro 2011, 31, 54-65. [CrossRef]

33. Yiu, B.Y.S.; Tsang, I.K.H.; Yu, A.C.H. GPU-based beamformer: Fast realization of plane wave compounding and synthetic aperture imaging. IEEE Trans. Ultrason. Ferroelectr. Freq. Control 2011, 58, 1698-1705. [CrossRef] [PubMed] 
34. Martin-Arguedas, C.J.; Romero-Laorden, D.; Martinez-Graullera, O.; Perez-Lopez, M.; Gomez-Ullate, L. An ultrasonic imaging system based on a new SAFT approach and a GPU beamformer. IEEE Trans. Ultrason. Ferroelectr. Freq. Control 2012, 59, 1402-1412. [CrossRef] [PubMed]

35. Hasegawa, H.; Kanai, H. High-frame-rate Echocardiography Using diverging transmit beams and parallel receive beamforming. J. Med. Ultrason. 2011, 38, 129-140. [CrossRef] [PubMed]

36. Papadacci, C.; Pernot, M.; Couade, M.; Fink, M.; Tanter, M. High contrast ultrafast imaging of the heart. IEEE Trans. Ultrason. Ferroelectr. Freq. Control 2014, 61, 288-301. [CrossRef] [PubMed]

37. Santos, P.; Haugen, G.U.; Løvstakken, L.; Samset, E.; D’hooge, J. Diverging Wave Volumentric Imaging Using Sub-aperture Beam forming. IEEE Trans. Ultrason. Ferroelectr. Freq. Control 2016, 63, 2114-2124. [CrossRef] [PubMed]

38. Cikes, M.; Tong, L.; Sutherland, G.R.; D’hooge, J. Ultrafast cardiac ultrasound imaging, Technical Principles, Applications and Clinical Benefits. JACC Cardiovasc. Imaging 2014, 7, 812-823. [CrossRef] [PubMed]

39. Prieur, F.; Denarie, B.; Austeng, A.; Torp, H. Multi-line transmission in medical imaging using the second-harmonic signal. IEEE Trans. Ultrason. Ferroelectr. Freq. Control 2013, 60, 2682-2692. [CrossRef] [PubMed]

40. Flaherty, J.J.; Erikson, K.R.; Lund, V.M. Synthetic Aperture Ultrasound Imaging Systems. U.S. Patent 3,548,642, 2 March 1967.

41. Burckhardt, C.B.; Grandchamp, P.A.; Hoffmann, H. An experimental $2 \mathrm{MHz}$ synthetic aperture sonar system intended for medical use. IEEE Trans. Ultrason. Ferroelectr. Freq. Control 1974, 21, 1-6. [CrossRef]

42. Ylitalo, J.T.; Ermert, H. Ultrasound Syntheric Aperture Imaging: Monostatic approach. IEEE Trans. Ultrason. Ferroelectr. Freq. Control 1994, 41, 333-339. [CrossRef]

43. Jensen, J.A.; Nikolov, S.I.; Gammelmark, L.; Pedersen, M.H. Synthetic Aperture Ultrasound Imaging. Ultrasonics 2006, 44, e5-e15. [CrossRef] [PubMed]

44. O'Donnel, M.; Thomas, L.J. Efficient Synthetic Aperture Imaging from a circular aperture with possible application to catheter-based imaging. IEEE Trans. Ultrason. Ferroelectr. Freq. Control 1992, 39, 366-380. [CrossRef] [PubMed]

45. Karaman, M.; Li, P.C.; O'Donnel, M. Synthetic aperture imaging for small scale systems. IEEE Trans. Ultrason. Ferroelectr. Freq. Control 1995, 42, 429-442. [CrossRef]

46. Karaman, M.; O'Donnel, M. Subaperture processing for ultrasonic imaging. IEEE Trans. Ultrason. Ferroelectr. Freq. Control 1998, 45, 126-135. [CrossRef] [PubMed]

47. Fredrik, G.; Jensen, J.A. Frequency Division Transmission Imaging and Synthetic Aperture Reconstruction. IEEE Trans. Ultrason. Ferroelectr. Freq. Control 2006, 53, 900-911.

48. Misaridis, T.X.; Gammelmark, K.; Jorgensen, C.H.; Lindberg, N.; Thomsen, A.H.; Pedersen, M.H.; Jensen, J.A. Potential of coded excitation in medical ultrasound imaging. Ultrasonics 2000, 38, 183-189. [CrossRef]

49. Misaridis, T.; Jensen, J.A. Use of modulated excitation signals inultrasound. Part I: Basic concepts and expected benefits. IEEE Trans. Ultrason. Ferroelectr. Freq. Control 2005, 52, 192-207. [CrossRef] [PubMed]

50. Gammelmark, K.L.; Jensen, J.A. Multielement synthetic transmit aperture imaging using temporal encoding. IEEE Trans. Med. Imaging 2003, 22, 552-563. [CrossRef] [PubMed]

51. Nikolov, S.I. Synthetic Aperture Tissue and Flow Ultrasound Imaging. Ph.D. Thesis, DTU Technical University of Denmark, Lyngby, Denmark, 2001.

52. Luchies, A.; Byram, B.C. Deep Neural Networks for Ultrasound Beamforming. IEEE Trans. Med. Imaging 2018, 37, 1464-1477. [CrossRef] [PubMed]

53. Savoia, A.S.; Matrone, G.; Ramalli, A.; Boni, E. Improved lateral resolution and contrast in ultrasound imaging using sidelobe masking technique. In Proceedings of the IEEE International Ultrasonics Symposium, Chicago, IL, USA, 3-6 September 2014.

54. Reeg, J.R.; Oelze, M.L. Improving lateral resolution in ultrasonic imaging by utilizing nulls in the beam pattern. In Proceedings of the IEEE International Ultrasonics Symposium, Taipei, Taiwan, 21-24 October 2015.

55. Reeg, J.R. Null Subtraction Imaging Technique for Biomedical Ultrasound Imaging. Master's Thesis, Electrical and Computer Engineering, Graduate College of the University of Illinois Urbana-Champaign, Champaign, IL, USA, 2016.

56. Lediju, M.; Trahey, G.E.; Byram, B.C.; Dahl, J.J. Short-lag spatial coherence of backscattered echoes: Imaging characteristics. IEEE Trans. Ultrason. Ferroelectr. Freq. Control 2011, 58, 1377-1388. [CrossRef] [PubMed] 
57. Dahl, J.J.; Hyun, D.; Lediju, M.; Trahey, G.E. Lesion detectability in diagnostic ultrasound with short-lag spatial coherence imaging. Ultrason. Imaging 2011, 33, 119-133. [CrossRef] [PubMed]

58. Dahl, J.J.; Hyun, D.; Li, Y.; Jakovljevic, M.; Bell, M.A.L.; Long, W.J.; Bottenus, N.; Kakkad, V.; Trahey, G.E. Coherence Beamforming and Its Applications to the Difficult-to-Image Patient. In Proceedings of the IEEE International Ultrasonics Symposium, Washington, DC, USA, 6-9 September 2017.

59. Jensen, J.A.; Holm, O.; Jensen, L.J.; Bendsen, H.; Nikolov, S.I.; Tomov, B.G.; Munk, P.; Hansen, M.; Salomonsen, K.; Hansen, J.; et al. Ultrasound research scanner for real-time synthetic aperture data acquisition. IEEE Trans. Ultrason. Ferroelectr. Freq. Control 2005, 52, 881-891. [CrossRef] [PubMed]

60. Jensen, J.A.; Holten-Lund, H.; Nilsson, R.T.; Hansen, M.; Larsen, U.D.; Domsten, R.P.; Tomov, B.G.; Stuart, M.B.; Nikolov, S.I.; Pihl, M.J.; et al. SARUS: A synthetic aperture real-time ultrasound system. IEEE Trans. Ultrason. Ferroelectr. Freq. Control 2013, 60, 1838-1852. [CrossRef] [PubMed]

61. Tortoli, P.; Bassi, L.; Boni, E.; Dallai, A.; Guidi, F.; Ricci, S. ULA-OP: An advanced open platform for ultrasound research. IEEE Trans. Ultrason. Ferroelectr. Freq. Control 2009, 56, 2207-2216. [CrossRef] [PubMed]

62. Boni, E.; Bassi, L.; Dallai, A.; Guidi, F.; Meacci, V.; Ramalli, A.; Ricci, S.; Tortoli, P. ULA-OP 256: A 256-channel open scanner for development and real-time implementation of new ultrasound methods. IEEE Trans. Ultrason. Ferroelectr. Freq. Control 2016, 63, 1488-1495. [CrossRef] [PubMed]

63. Lewandowski, M.; Walczak, M.; Witek, B.; Kulesza, P.; Sielewicz, K. Modular \& scalable ultrasound platform with GPU processing. In Proceedings of the 2012 IEEE International Ultrasonics Symposium, Dresden, Germany, 7-10 October 2012.

64. Cheung, C.; Yu, A.; Salimi, N.; Yiu, B.; Tsang, I.; Kerby, B.; Azar, R.; Dickie, K. Multi-channel pre-beamformed data acquisition system for research on advanced ultrasound imaging methods. IEEE Trans. Ultrason. Ferroelectr. Freq. Control 2012, 59, 243-253. [CrossRef] [PubMed]

(C) 2018 by the author. Licensee MDPI, Basel, Switzerland. This article is an open access article distributed under the terms and conditions of the Creative Commons Attribution (CC BY) license (http://creativecommons.org/licenses/by/4.0/). 